\title{
Early Oncological and Functional Outcomes of Prostate Capsule Sparing Cystectomy Compared with Standard Radical Cystectomy
}

\author{
Ahmed Y. Abdelaziz ${ }^{\mathrm{a}}$ Hossam Shaker ${ }^{\mathrm{b}}$ Mohamed Seifelnasr ${ }^{\mathrm{b}}$ Hossam Elfol ${ }^{\mathrm{c}}$ \\ Mohamed Nazim ${ }^{d} \quad$ Mohamed Mahmoued ${ }^{b}$ \\ aUrology Department, Faculty of Medicine, Cairo University, Cairo; ' ${ }^{b}$ rology Department; ' Oncology Department, Faculty of \\ Medicine, Fayoum University, Fayoum; dUrology Department, Faculty of Medicine, Alazhar University, Cairo, Egypt
}

\begin{abstract}
Key Words
Prostate sparing $\cdot$ Radical cystectomy • Continence $•$ Erectile dysfunction $\cdot$ Bladder cancer
\end{abstract}

\begin{abstract}
Introduction and Objectives: A comparative study of standard radical cystectomy and prostate capsule sparing radical cystectomy regarding functional and oncological outcomes. Materials and Methods: A randomized study of 96 patients with transitional cell carcinoma of the bladder (December 2014 - June 2016) was done. We excluded cases with preoperative T4 staging, lymphadenopathy, prostatic specific antigen $>4 \mathrm{ng} / \mathrm{dl}$, and cases with positive biopsies from the bladder neck, trigone, and/or prostatic urethra. Patients were divided into 2 groups, Group 1: standard radical cystectomy with orthotopic diversion ( $n=51)$, Group 2: prostate capsule sparing cystectomy with orthotopic diversion $(n=45)$. Preoperative transrectal ultrasound and prostatic biopsies were done in Group 2 to exclude prostate cancer. We compared the urinary continence and erectile function in both groups after 6 months, 1, and 2 years. Results: There was no significant difference between the groups regarding preoperative demographic data, tumor stage, grade, site by cystoscopy, and biopsy. Intraoperative monitoring showed no significant differences regarding blood loss, surgical complications, or operative time ( $2.5 \pm 0.48$ vs. $2.4 \pm 0.45 \mathrm{~h})$. There was a significantly higher percentage of continence and po-
\end{abstract}

\section{KARGER}

Fax +4161306 1234

E-Mailkarger@karger.com

www.karger.com
(C) 2019 The Author(s)

Published by S. Karger AG, Basel Open access

This article is licensed under the Creative Commons AttributionNonCommercial-NoDerivatives 4.0 International License (CC BYNC-ND) (http://www.karger.com/Services/OpenAccessLicense). Usage and distribution for commercial purposes as well as any distribution of modified material requires written permission. tency in Group 2 than in Group 1. Sixteen cases (35.6\%) in Group 2 but only 4 cases (7.8\%) in Group 1 developed large post-voiding residual urine and needed intermittent selfcatheterization cleaning ( $p=0.001$ ). The tumor recurrence rate was not significantly different between the groups after 2 years $(p=0.3)$. Conclusion: Prostate capsule sparing cystectomy is a good option in selected cases with better continence and potency and without compromising oncological outcomes after 2 years.

(c) 2019 The Author(s)

Published by S. Karger AG, Basel

\section{Introduction}

Urothelial carcinoma is the most common malignancy of the urinary tract and is the second most common cause of death among genitourinary tumors [1]. It accounts for $7 \%$ of new cancer cases in men and $2 \%$ of new cancer cases in women. The average age at time of diagnosis is 65 years. Approximately $75 \%$ of bladder cancers are localized to the bladder at the time of presentation, and $25 \%$ have spread to regional lymph nodes (LNs) or distant sites [2].

Radical cystectomy includes removal of the anterior pelvic organs which in men includes the bladder with its surrounding fat and peritoneal attachments, the prostate, and the seminal vesicles. This is considered the gold

Ahmed Yehia Abdelaziz

Urology Department, Faculty of Medicine Cairo University

EG-11562 Cairo (Egypt)

E-Maildr_ahmedyehia81@yahoo.com 
standard of treatment for muscle invasive bladder cancer [2]. Modified cystectomy includes removal of the bladder, pelvic LN dissection, and sparing seminal vesicles, vas deferens, and the prostate (apical part or prostatic capsule) and neobladder substitution without use of cautery near neurovascular bundles [3].

Focusing on functional outcomes, modified cystectomy provides better potency and continence (especially night time continence) compared to radical cystectomy [4-6]. Modified cystectomy is performed to improve postoperative continence, potency, and sometimes even fertility especially in young patients with bladder cancer [7], but other studies reported a higher oncological failure [8]. Our study aimed to evaluate the oncological and functional outcome of prostate capsule sparing cystectomy (PCSC) in comparison to standard radical cystectomy.

\section{Methods}

This study included 154 patients who presented to the Urology Department, Fayoum University with transitional cell carcinoma (TCC) of the bladder candidate for radical cystectomy in the period from December 2014 to June 2016. This is a prospective randomized study in which patients were randomly divided into 2 equal groups by simple randomization using computer generated random numbers by Microsoft Excel.

The cases were divided into 2 groups: Group 1 was subjected to standard radical cystectomy and Group 2 was subjected to PCSC. A written informed consent was obtained from all patients. The study protocol, as well as the suggested informed consent, were approved by the Institutional Review Board of the Urology Department before enrolling participants.

All patients presented in our center with bladder masses. Patients were assessed with a full medical and surgical history, a general and abdominal examination including a digital rectal examination, and CT or MRI of the abdomen and pelvis with oral and intravenous contrast. Cystoscopy was done in all cases and biopsies were taken from the bladder mass, bladder trigone, bladder neck, and prostatic urethra.

All the included cases were assessed preoperatively with routine full labs (kidney functions, liver functions, complete blood count, bleeding profile, and serum glucose), urine analysis and culture, total and free prostatic specific antigen (PSA), full cardiological assessment in the form of electrocardiography, echocardiography, management of any cardiological diseases, and assessment of the chest and hepatic condition. We used Charlson comorbidities score for assessment of all patients preoperatively [9]. Diabetes and hypertension were preoperatively controlled. All cases were preoperatively urinary continent. Erectile function was assessed in all cases using the International Index of Erectile Function Questionnaire (IIEF-5) [11]. The questionnaire classifies erectile dysfunction (ED) severity into 5 categories: severe (5-7), moderate (8-11), mild to moderate (12-16), mild (17-21), and no ED (22-25).
Prostatic biopsies were taken preoperatively in all cases of Group 2 to exclude prostate cancer (PCa). A 12-core biopsy according to American Urological Association was conducted under ultrasound guidance by a transrectal approach [10].

In our study, we excluded cases with tumors other than TCC, suspected high stage (cT4) tumors, cases with positive LN, and positive distant metastasis in preoperative investigations, cases with positive biopsies from the bladder neck, trigone, and/or prostatic urethra, cases with PSA $>4 \mathrm{ng} / \mathrm{dl}$ in Group 1 or with positive prostatic biopsy in Group 2, and cases with a high Charlson comorbidities score who were medically unfit for surgical intervention [9].

All operations in both groups were done by the oncology team (2 surgeons and 2 assistants) of the Urology Department in AlFayoum University. Standard radical cystectomy and neobladder substitution was done in Group 1 with surgical removal of the bladder and its coverings, prostate, seminal vesicles, vas deferens and radical lymphadenectomy of pelvic LN up to the common iliac lymph nodes, and neobladder substitution (Studer pouch) using the ileal intestinal segment.

Modified PCSC was done in Group 2. The primary goal of PCSC was to remove the bladder while preserving the external urethral sphincter and avoid damaging the neurovascular bundles that contribute to sexual function. The antegrade plane of dissection was performed until the endopelvic fascia was laterally reached and the seminal vesicles were medially identified. Following antegrade dissection, the endopelvic fascia was opened on either side of the prostate. A single absorbable suture line was placed parallel to the bladder neck similar to a Millin retropubic prostatectomy [12]. The prostate capsule was then entered proximal to the suture line. The prostate adenoma was separated from the capsule using a combination of blunt and sharp dissection. The urethra was identified and divided and the adenoma was separated from the posterior prostate capsule. The posterior bladder neck was identified and divided removing the bladder and prostate adenoma intact and leaving the prostate capsule/peripheral zone in situ. An extended LN dissection was performed bilaterally to the level of the common iliac LN.

A neobladder (Studer pouch) was then fashioned from the terminal ileal intestinal segment $(40 \mathrm{~cm})$ in all patients of both groups. The neobladder was anastomosed to the prostate peripheral zone/ capsule using absorbable sutures in a running fashion first closing the posterior anastomosis. Ureteral stents were brought through the neobladder wall and the bladder was drained with a $20 \mathrm{~F}$ Foley catheter. Stents were removed on the 7 th or 8th day and urethral catheters were removed on day 14 postoperatively.

Intraoperative data included monitoring for intraoperative complications, operative time, and the amount of blood loss.

\section{Follow-Up Protocol}

After removal of the ureteric stents and urethral catheter all patients were followed-up as follow:

- A complete physical examination, kidney functions, and serum electrolytes were done at 6 months, 1 , and 2 years.

- Postoperative abdominopelvic ultrasound was used to measure the post-voiding residual urine at 6 months, 1 , and 2 years.

- Continence and potency were assessed at 6 months, 1, and 2 years.

- We considered that the patients were continent when they were dry during the day or at night without need of any pads. 


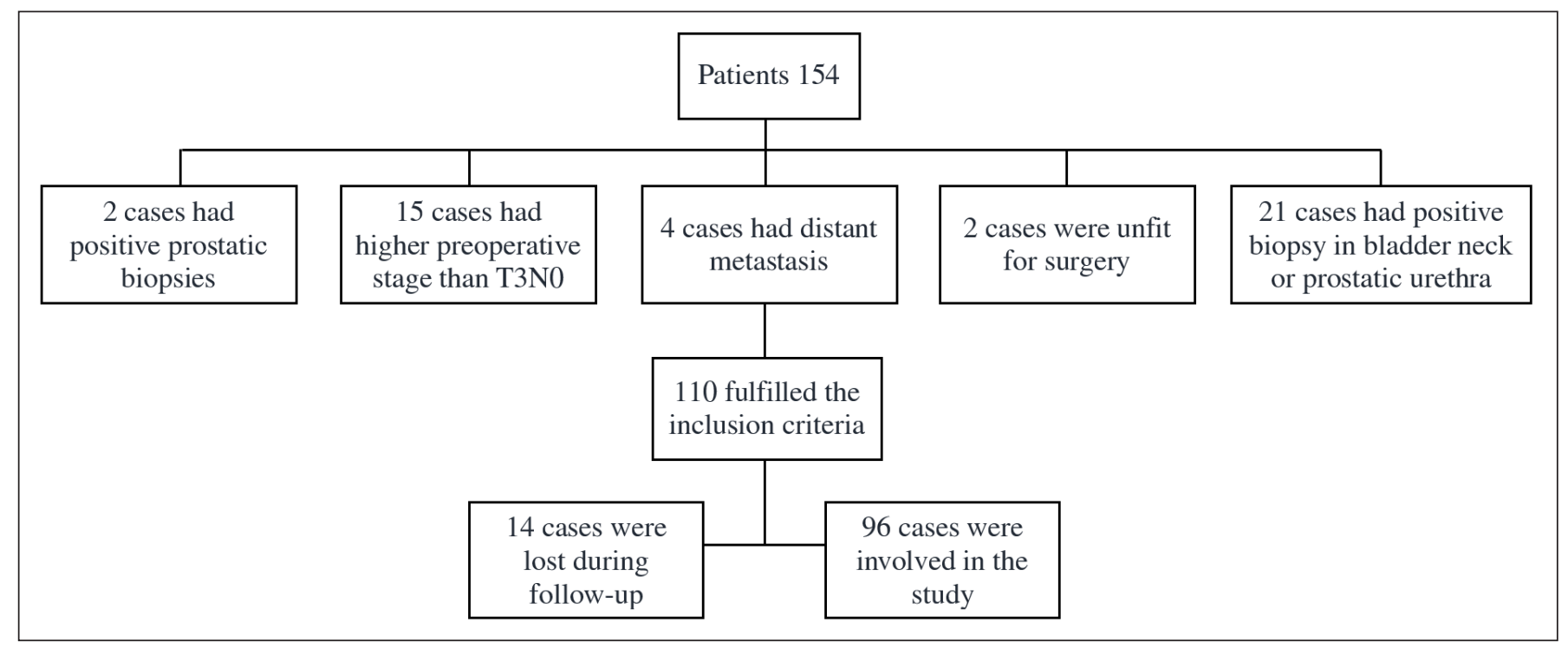

Fig. 1. Number of patients involved in the study.

Table 1. Preoperative demographic and tumor characteristics

\begin{tabular}{llll}
\hline & Group 1 & Group 2 & $\mathrm{p}$ \\
& & & \\
\hline Age, years & $63.7 \pm 6.8$ & $62.2 \pm 5.8$ & 0.07 \\
BMI, kg/m² & $23.3 \pm 2.3$ & $24.1 \pm 2.1$ & 0.6 \\
Preoperative creatinine & $1.2 \pm 0.37$ & $1.1 \pm 0.33$ & 0.1 \\
Tumor stage, n (\%) & & & 0.4 \\
T1 & $13(25.5 \%)$ & $13(28.9 \%)$ & \\
T2 & $38(74.5 \%)$ & $32(71.1 \%)$ & \\
Tumor grade, n (\%) & & & 0.2 \\
Low & $13(25.5 \%)$ & $18(40 \%)$ & \\
High & $38(74.5 \%)$ & $27(60 \%)$ & \\
Tumor site, n (\%) & & & 0.2 \\
$\quad$ Anterior wall & $6(11.8 \%)$ & $9(20 \%)$ & \\
Posterior wall & $9(17.6 \%)$ & $2(4.4 \%)$ & \\
Left lateral wall & $15(29.4 \%)$ & $9(20 \%)$ & \\
Right lateral wall & $15(29.4 \%)$ & $17(37.8 \%)$ & \\
Dome & $6(11.8 \%)$ & $8(17.8 \%)$ & \\
& &
\end{tabular}

$\mathrm{BMI}=$ Body mass index .

- Erectile function was assessed using the IIEF before and after modified PCSC and standard cystectomy.

- CT of the abdomen and pelvis with oral and intravenous contrast was done every 6 months postoperatively to exclude local recurrence.

Comparison between Prostate Sparing and Radical Cystectomy
Statistical Analysis

Data were collected and coded to facilitate data manipulation and double entered into Microsoft Access and data analysis was performed using SPSS software version 18 in Windows 7.

Simple descriptive analysis was in the form of numbers and percentages for qualitative data and arithmetic means as a central tendency measurement, standard deviations as a measure of dispersion for quantitative parametric data, and an inferential statistic test.

For quantitative parametric data, the independent student t-test was used to compare measures of 2 independent groups of quantitative data. A paired t-test compared 2 dependent quantitative data.

For qualitative data, the Chi square test compared 2 or more than 2 qualitative groups. The McNemar test was used for paired dependent qualitative data. The general linear model was used to compare repeated measures. A p-value $\leq 0.05$ was considered the cut-off value for significance.

\section{Results}

Our study included 96 cases of 154 male patients with TCC of the bladder as 42 cases were excluded as they did not fulfill the inclusion criteria, 2 cases were excluded from Group 2 as preoperative prostatic biopsies were positive for $\mathrm{PCa}$, and 14 cases were lost during the follow-up (fig.1). Of the 14 patients who were lost during follow-up, 6 patients did not respond to our follow-up protocol and 8 patients died during the follow-up period.

Curr Urol 2019;13:37-45 
Table 2. Postoperative urinary continence and erectile functions improvement

\begin{tabular}{|c|c|c|c|}
\hline & Group 1, n (\%) & Group 2, n (\%) & $\mathrm{p}$ \\
\hline \multicolumn{4}{|l|}{ Continence } \\
\hline After 6 months & & & $<0.001$ \\
\hline Incontinent & $25(49 \%)$ & $2(4.4 \%)$ & \\
\hline Dryness in day and night & $17(33.3 \%)$ & $39(86.7 \%)$ & \\
\hline Dryness day only & $9(17.6 \%)$ & $4(8.9 \%)$ & \\
\hline After 1 year & & & $<0.001$ \\
\hline Incontinent & $25(49 \%)$ & $2(4.4 \%)$ & \\
\hline Dryness in day and night & $19(37.3 \%)$ & $39(86.7 \%)$ & \\
\hline Dryness day only & $7(13.7 \%)$ & $4(8.9 \%)$ & \\
\hline After 2 years & & & $<0.001$ \\
\hline Incontinent & $24(47.1 \%)$ & $1(2.2 \%)$ & \\
\hline Dryness in day and night & $22(43.1 \%)$ & $41(91.1 \%)$ & \\
\hline Dryness day only & $5(9.8 \%)$ & $3(6.7 \%)$ & \\
\hline \multicolumn{4}{|l|}{ - } \\
\hline Preoperative & & & 0.2 \\
\hline No ED & $40(78.4 \%)$ & $41(91.1 \%)$ & \\
\hline Mild ED & $10(19.6 \%)$ & $3(6.7 \%)$ & \\
\hline Moderate ED & $1(2 \%)$ & $1(2.2 \%)$ & \\
\hline 6 months postoperatively & & & $<0.001$ \\
\hline No ED & $0(0 \%)$ & $40(88.9 \%)$ & \\
\hline Mild ED & $2(3.9 \%)$ & $2(4.4 \%)$ & \\
\hline Moderate ED & $4(7.8 \%)$ & $2(4.4 \%)$ & \\
\hline Severe ED & $45(88.2 \%)$ & $1(2.2 \%)$ & \\
\hline 1 year postoperatively & & & $<0.001$ \\
\hline No ED & $0(0 \%)$ & $40(88.9 \%)$ & \\
\hline Mild ED & $2(3.9 \%)$ & $2(4.4 \%)$ & \\
\hline Moderate ED & $4(7.8 \%)$ & $2(4.4 \%)$ & \\
\hline Severe ED & $45(88.2 \%)$ & $1(2.2 \%)$ & \\
\hline 2 years postoperatively & & & $<0.001$ \\
\hline No ED & $0(0 \%)$ & $40(88.9 \%)$ & \\
\hline Mild ED & $2(3.9 \%)$ & $2(4.4 \%)$ & \\
\hline Moderate ED & $4(7.8 \%)$ & $2(4.4 \%)$ & \\
\hline Severe ED & $45(88.2 \%)$ & $1(2.2 \%)$ & \\
\hline
\end{tabular}

There was no significant difference between the 2 groups regarding preoperative demographic, laboratory data, tumor stage, grade and site by cystoscopy, and biopsy (table 1).

Intraoperative monitoring for all cases showed that there was no significant difference between the 2 groups regarding blood loss or surgical complications, where there were no significant intraoperative surgical complications in the cases of both groups.

There was also no significant difference regarding operative time where the mean time for cystectomy without diversion in Group 1 was $2.5 \pm 0.48$ hours while in Group 2 it was $2.4 \pm 0.45$ hours.
Postoperatve complications in both groups were of Grade 1 and Grade 2 according to the Clavien-Dindo classification with no significant difference between the groups [13]. Postoperative specimen pathology showed no significant difference regarding $\mathrm{T}$ stage, $\mathrm{N}$ stage, or tumor size. The pathologies of the prostates in all cases were free from $\mathrm{PCa}$. Postoperative pathology showed TCC in the prostatic urethra in 8 cases of Group 1.

Preoperatively, all patients were urinary continent. We compared the urinary continence in both groups after 6 months, 1 , and 2 years. We considered the patients continent when they were dry without needing any pads during night or day. We found that there was a significant 
Table 3. Erectile function follow-up among both groups

\begin{tabular}{|c|c|c|c|c|}
\hline Preoperative & 6 months & 1 year & 2 years & $\mathrm{p}$ \\
\hline \multicolumn{5}{|c|}{ Group 1} \\
\hline $10(19.6 \%)$ & $2(3.9 \%)$ & $2(3.9 \%)$ & $2(3.9 \%)$ & \\
\hline $1(2 \%)$ & $4(7.8 \%)$ & $4(7.8 \%)$ & $4(7.8 \%)$ & \\
\hline $0(0 \%)$ & $45(88.2 \%)$ & $45(88.2 \%)$ & $45(88.2 \%)$ & \\
\hline $41(91.1 \%)$ & $40(88.9 \%)$ & $40(88.9 \%)$ & $40(88.9 \%)$ & \\
\hline $3(6.7 \%)$ & $2(4.4 \%)$ & $2(4.4 \%)$ & $2(4.4 \%)$ & \\
\hline $1(2.2 \%)$ & $2(4.4 \%)$ & $2(4.4 \%)$ & $2(4.4 \%)$ & \\
\hline $0(0 \%)$ & $1(2.2 \%)$ & $1(2.2 \%)$ & $1(2.2 \%)$ & \\
\hline
\end{tabular}

Table 4. Postoperative residual urine and recurrence

\begin{tabular}{lccc}
\hline & Group 1 & Group 2 & $\mathrm{p}$ \\
\hline $\begin{array}{l}\text { Large residual urine } \\
(>300 \mathrm{ml}) \text { and CISC }\end{array}$ & & 0.001 \\
$\quad$ Yes & $4(7.8 \%)$ & $16(35.6 \%)$ & \\
No & $47(92.2 \%)$ & $29(64.4 \%)$ & \\
\hline
\end{tabular}

difference between the 2 groups with a higher percentage of total urinary incontinence among Group 1 (about $47.1 \%$ at 2 years) versus a higher percentage of dryness during day and night (about $91.1 \%$ at 2 years) in Group 2. There was no significant difference in urinary incontinence among each group during follow-up. There was no statistically significant difference between the groups regarding preoperative ED. On the other hand there was a statistically significant difference in ED follow-up between them with a high percentage of severe ED (88.2\%) at 2 years among Group 1 versus a high percentage of no ED (88.9\%) at 2 years among Group 2 (table 2).

There was a significant decrease in erectile function postoperatively in Group 1 as $88.2 \%$ developed severe ED 6 months postoperatively and continued until 2 years in the follow-up, but there was no postoperative significant difference in Group 2 which indicated that PCSC keeps the erectile function of the patients postoperatively (table 3).

Sixteen cases $(35.6 \%)$ in Group 2 but only 4 cases (7.8\%) in Group 1 developed large post-voiding residual

Comparison between Prostate Sparing and

Radical Cystectomy urine $(>300 \mathrm{ml})$ and needed intermittent self-catheterization cleaning (CISC) with a statistically significant difference (table 4).

Eight patients (five in Group 1 and three in Group 2) died during the follow-up period and were excluded from the study. In Group 1, 2 patients died from the tumor and 3 died of other causes ( 2 cases of pulmonary embolism and 1 case of myocardial infarction). In Group 2, 1 patient died from the tumor and 2 cases died because of other causes (one of hepatic failure and the other of pulmonary embolism).

There was no significant differences between the 2 groups regarding the 2-year survival rate as it was $91 \%$ in Group 1 and $93.75 \%$ in Group 2 (table 5).

There was no statistically significant difference between different types of cystectomy as regards mean duration of survival and the rate of tumor recurrence, which indicated that both types of cystectomy have the same survival duration and recurrence rate (table 5) (fig. 2).

\section{Discussion}

The treatment of choice for muscle invasive and refractory cases of superficial high grade bladder cancer is radical cystectomy. It is usually associated with significant morbidity in urinary and sexual functions as the pelvic nerves and to a lesser extent the external sphincter are usually damaged during deep pelvic dissection.

PCSC represents another alternative to standard radical cystectomy for preservation of urinary and sexual functions. Despite these promising functional results, 


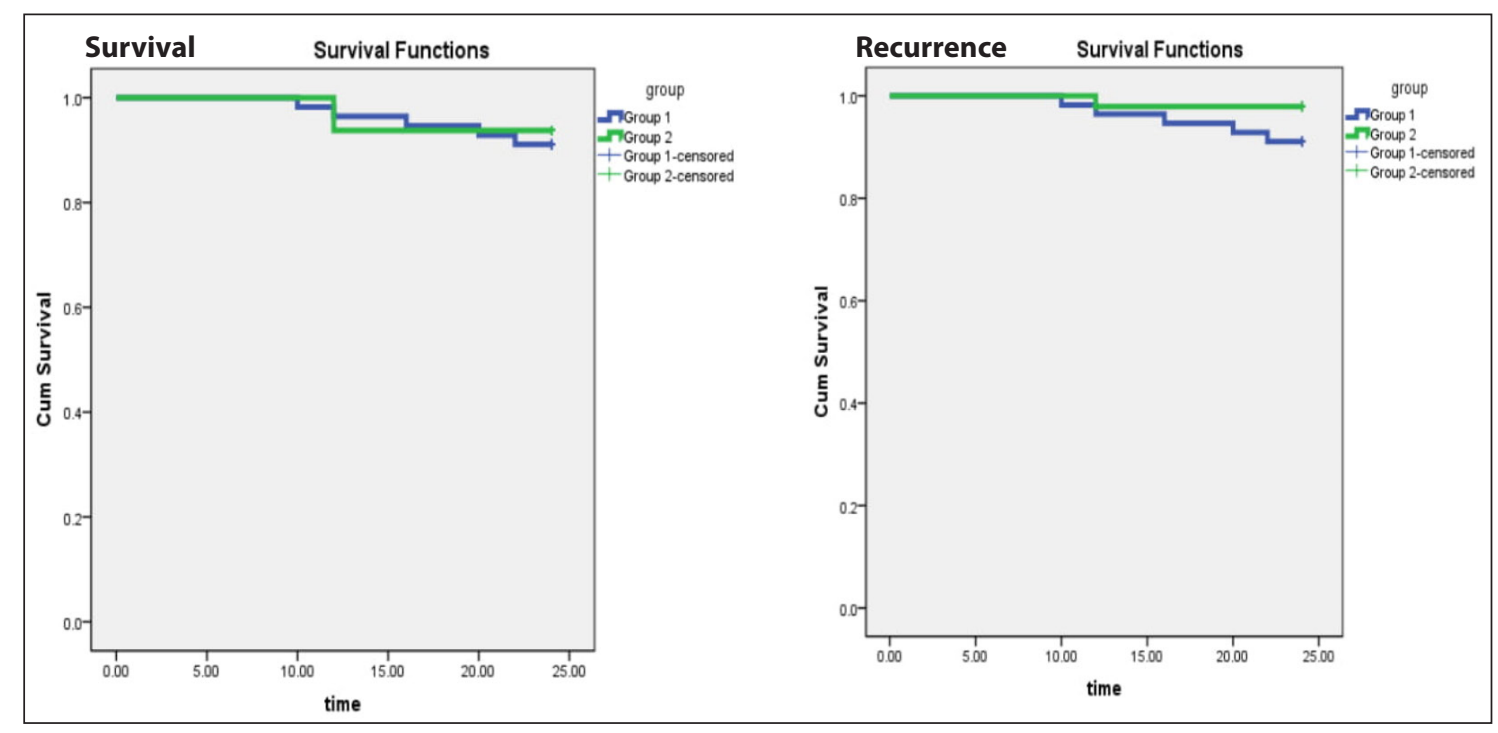

Fig. 2. Comparisons of survival and recurrence rate in different types of cystectomy.

Table 5. Comparisons of survival and recurrence rate in different types of cystectomy

\begin{tabular}{|c|c|c|c|c|c|c|c|}
\hline & \multicolumn{3}{|c|}{ Group $1(n=56)$} & \multicolumn{3}{|c|}{ Group $2(n=48)$} & \multirow[b]{2}{*}{$\mathrm{p}$} \\
\hline & No. of event & Mean & SE & No. of event & Mean & SE & \\
\hline Survival & $5(8.9 \%)$ & 23.3 & 0.36 & $3(6.3 \%)$ & 23.3 & 0.42 & 0.62 \\
\hline Relapse survival & $5(8.9 \%)$ & 23.3 & 0.36 & $1(2.1 \%)$ & 23.7 & 0.25 & 0.15 \\
\hline
\end{tabular}

concerns over residual $\mathrm{PCa}$, local recurrence, and metastatic disease necessitate appropriate selection of the patient for this procedure $[4,8]$.

PCSC is considered as a less radical surgery so there is a risk of leaving urothelial carcinoma or PCa behind, both of which would be difficult to treat. We excluded all cases with positive prostatic urethral biopsies in both groups preoperativelly and our study reported that only 8 patients $(15 \%)$ had urothelial carcinoma involving the prostatic urethra in the postoperative pathology of Group 1.

The incidence of prostatic involvement by TCC in cystectomy specimens in unscreened patients is $20-48 \%$. Revelo et al. [14] found $48 \%$ of cases has prostatic involvement by TCC in cystectomy specimens. Wood et al. [15] performed step sections on 84 specimens of radical cystectomy. They found that $43 \%$ of patients had TCC involving the prostate including $17 \%$ with stromal involve- ment. However, their study demonstrated that preoperative biopsy can identify patients with TCC involving the prostate. Revelo et al. [14] performed step sections on 121 patients in order to detect whether the prostate apex could be spared to improve continence in patients undergoing radical cystectomy and neobaldder. They identified $58(48 \%)$ men with TCC of the prostate including 16 patients (13\%) involving the apex of the prostate.

\section{Oncological Outcome}

The major concern of PCSC is the potential risk of postoperative local recurrence and metastatic disease. Our study reported only 5 cases of local recurrence in Group 1, 1 case in Group 2, and no distant metastases in either group after 2 years with no significant difference. Several series with at least 3 years follow-up have reported low rates of local recurrence that are similar to those found in standard radical cystectomy [4-6, 16-21]. 
Mertens et al. [21] studied 110 patients with cT14NO-3 bladder tumors treated with PCSC and they concluded that it is an oncologically safe procedure with good functional outcome. Their results showed that 2and 5-year recurrence-free survival rates were 71.2 and $66.6 \%$, and distant and local recurrence rates were 34.2 and $10.0 \%$, respectively in a median follow-up period of 77 months. Complete daytime and nighttime dryness occurred in 96.2 and $81.9 \%$ of patients, respectively, and erectile function was intact in $89.7 \%$.

Botto et al. [17] found an increased risk of distant metastasis $(17.6 \%)$ in patients undergoing PCSC without local recurrence suggesting the presence of micrometastatic disease prior to intervention. In another study of 100 patients undergoing PCSC, 5 cases developed local recurrence and 31 patients developed distant metastases, but of these patients, 17 had T3 disease and/or nodal disease suggesting that it was the stage and not the procedure driving the outcome [4]. Hautmann et al. [8] did not recommend PCSC as they reported a $10-15 \%$ higher oncological failure rate in the form of local and distant recurrence.

Our patients were carefully preoperatively assessed to exclude the possibility of associated PCa. Prostatic biopsies were taken in all cases in Group 2 and we excluded cases with PSA $>4 \mathrm{ng} / \mathrm{dl}$ in Group 1 . The rate of incidentally discovered $\mathrm{PCa}$ in specimens of radical cystectomy is variable, ranging from $4 \%$ in Lee et al. [22] series to $60 \%$ in Winkler et al. [23] series. In our study, none of the patients in Group 2 developed PCa in the 2 years follow-up and there was no PCa detected in any specimen of radical cystectomy in either group. Some studies detected incidental PCa in postoperative specimens and they did not give any additional therapy as it was a low risk PCa. They followed these patients with periodic PSA and digital rectal examination. The definition of PCa progression in patient post-PCSC is based in the PSA nadir archived for each patient, as the criteria of two consecutive values of $0.2 \mathrm{ng} / \mathrm{ml}$ or more cannot be used because part of the prostate is left [24]. Many studies showed no PCa developed after PCSC $[6,16,19]$. Other studies showed a very low incidence $(2-4 \%)$ of PCa in these patients $[4,17,19,20,25]$.

\section{Functional Outcomes}

The main goal of PCSC is to improve the functional outcome without compromising the disease control. Unlike standard radical cystectomy, PCSC avoids dissection of the posterolateral aspect of the prostate, and the manipulation of the seminal vesicles. This preserves the

Comparison between Prostate Sparing and

Radical Cystectomy neurovascular bundles and avoids their injury resulting in preservation of potency in men with an adequate baseline sexual function [16].

In our study, although there was no significant difference in the preoperative baseline sexual function, $88 \%$ of Group 1 developed severe ED while no postoperative significant changes occurred in Group 2. These results are similar to many previous studies on PCSC $[4,5,16-$ $18,20,21,24]$.

Although urinary continence is an important goal in radical cystectomy with orthotopic diversion, daytime or nighttime incontinence are common after this procedure. This may be due to injury of the external urethral sphincter during deep dissection or injury of neurovascular bundles and so PCSC may give better results [18].

The literature on PCSC used various measures to evaluate urinary continence including questionnaires, voiding diaries, urodynamic studies, and pad tests $[4,16,18]$. In our study we considered the pad test as a measure for urinary continence. Our study showed that Group 2 were significantly drier than Group 1. Forty three percent of Group 1 versus $91 \%$ of Group 2 were dry during the daytime and nighttime after 2 years.

These results are similar to many studies, as most studies report daytime continence rates of $60-100 \%$ with most series reporting continence in the $90 \%$ plus range $[4,18,20]$. Colombo et al. [18] reported a $100 \%$ daytime continence rate 15 days after catheter removal. The rates of nighttime continence are more variable. While most studies report continence rates between 80 and $100 \%$ [4, $16,19,20,24]$, Muto et al. [5] reported a nighttime continence rate of only $31 \%$.

We noted a higher incidence of large post voiding residual urine (> $300 \mathrm{ml}$ ) and CISC in Group 2 (35.6\%) than in Group $1(7.8 \%)$. Colombo et al. [18] found the same results as postoperative urodynamic evaluation revealed adequate neobladder volumes of low pressure with high outlet resistance and they advised the patients who cannot generate an adequate Valsalva or adequately relax their pelvic floor for CISC. Nieuwenhuijzen et al. [20] also demonstrated that the International Prostate Symptoms Score was higher in these patients postoperatively likely due to the need to strain to complete micturition.

Kefer et al. [26] in their series concluded that despite that PCSC improves postoperative continence and the potency rate and gives excellent postoperative functional results, many concerns have been raised regarding the oncological outcme because most of the literature offers limited long-term outcomes. They recommended proper selection of these patients and defined a subgroup of pa-

Curr Urol 2019;13:37-45 
tients for whom the risk/benefit ratio of this procedure is potentially favorable.

There are some limitations in our study such as the small number of cases. We need to study a larger number of patients. A 2-year follow-up is considered a short period to evaluate the oncological outcome of PCSC, and we need a longer follow-up for these patients.

\section{Acknowledgement}

I would like to express my sincere gratitude to the urology departement and university faculty, Fayoum University and its chairman for support and help.

\section{Conclusion}

PCSC is a good option in selected cases with better continence and potency and without compromising oncological outcomes at 2 years but with a higher incidence of large residual urine.

\section{References}

-1 Pasin E, Josephson DY, Mitra AP, Cote RJ, Stein JP: Superficial bladder cancer: an update on etiology, molecular development, classification, and natural history. Rev Urol 2008; 10:31-43.

2 Konety BR, Carroll PR: Urothelial carcinoma: cancers of the bladder, ureter, and renal pelvis; in McAninch JW, Lue TF (eds): Smith and Tanagho's General Urology, ed 18. McGraw-Hill, 2013.

$\checkmark 3$ Klotz L, Pinthus J: The case for prostate capsule-sparing radical cystectomy in selected patients. Can Urol Assoc J 2009;3(6 Suppl 4):S215-S219.

4 Vallancien G, Abou El Fettouh H, Cathelineau X, Baumert H, Fromont G, Guillonneau B: Cystectomy with prostate sparing for bladder cancer in 100 patients: 10-year experience. J Urol 2002;168:2413-2417.

$\checkmark 5$ Muto G, Bardari F, D’Urso L, Giona C: Seminal sparing cystectomy and ileocapsuloplasty: long-term followup results. J Urol 2004; 172:76-80.

-6 Terrone C, Cracco C, Scarpa RM, Rossetti SR: Supra-ampullar cystectomy with preservation of sexual function and ileal orthotopic reservoir for bladder tumor: twenty years of experience. Eur Urol 2004;46:264-270.

$\checkmark 7$ Richards KA, Parks GE, Badlani GH, Kader AK, Hemal AK, Pettus JA: Developing selection criteria for prostate-sparing cystectomy: a review of cystoprostatectomy specimens. Urology 2010;75:1116-1120.
8 Hautmann RE, Stein JP: Neobladder with prostatic capsule and seminal-sparing cystectomy for bladder cancer: a step in the wrong direction. Urol Clin North Am 2005;32:177185.

$\checkmark 9$ Charlson ME, Pompei P, Ales KL, MacKenzie CR: A new method of classifying prognostic comorbidity in longitudinal studies: development and validation. J Chronic Dis 1987;40:373-383.

-10 Bjurlin MA, Carter HB, Schellhammer P, Cookson MS, Gomella LG, Troyer D, Wheeler TM, Schlossberg S, Penson DF, Taneja SS: Optimization of initial prostate biopsy in clinical practice: sampling, labeling and specimen processing. J Urol 2013; 189:2039-2046.

11 Rosen R, Riley A, Wagner G, Osterloh IH, Kirkpatrick J, Mishra A: The international index of erectile function (IIEF): a multidimensional scale for assessment of erectile dysfunction. Urology 1997;49:822-830.

12 Millin T: Retropubic prostatectomy: a new extravesical technique report on 20 cases.1945. J Urol 2002;167:976-979.

13 Dindo D, Demartines N, Clavien PA: Classification of surgical complications: a new proposal with evaluation in a cohort of 6336 patients and results of a survey. Ann Surg 2004;240:205-213.
14 Revelo MP, Cookson MS, Chang SS, Shook MF, Smith JA Jr, Shappell SB: Incidence and location of prostate and urothelial carcinoma in prostates from cystoprostatectomies: implications for possible apical sparing surgery. J Urol 2004;171:646-651.

15 Wood DP Jr, Montie JE, Pontes JE, Levin HS: Identification of transitional cell carcinoma of the prostate in bladder cancer patients: a prospective study. J Urol 1989;142:83-85.

16 Martis G, D’Elia G, Diana M, Ombres M, Mastrangeli B: Prostatic capsule- and nerve-sparing cystectomy in organ-confined bladder cancer: preliminary results. World J Surg 2005;29:1277-1281.

-17 Botto H, Sebe P, Molinie V, Herve JM, Yonneau L, Lebret T: Prostatic capsule- and seminal-sparing cystectomy for bladder carcinoma: initial results for selected patients. BJU Int 2004;94:1021-1025.

- 18 Colombo R, Bertini R, Salonia A, Naspro R, Ghezzi M, Mazzoccoli B, Deho' F, Montorsi F, Rigatti P: Overall clinical outcomes after nerve and seminal sparing radical cystectomy for the treatment of organ confined bladder cancer. J Urol 2004;171:1819-1822.

$>19$ Arroyo C, Andrews H, Rozet F, Cathelineau $\mathrm{X}$, Vallancien G: Laparoscopic prostate-sparing radical cystectomy: the Montsouris technique and preliminary results. $\mathrm{J}$ Endourol 2005; 19:424-428. 
20 Nieuwenhuijzen JA, Meinhardt W, Horenblas S: Clinical outcomes after sexuality preserving cystectomy and neobladder (prostate sparing cystectomy) in 44 patients. J Urol 2005;173:1314-1317.

21 Mertens LS, Meijer RP, de Vries RR, Nieuwenhuijzen JA, van der Poel HG, Bex A, van Rhijn BW, Meinhardt W, Horenblas S: Prostate sparing cystectomy for bladder cancer: 20-year single center experience. J Urol 2014;191:1250-1255.
22 Lee SH, Chang PL, Chen SM, Sun GH, Chen CL, Shen BY, Wu YS, Tsui: Synchronous primary carcinomas of the bladder and prostate. Asian J Androl 2006;8:357-359.

23 Winkler MH, Livni N, Mannion EM, Hrouda D, Christmas T: Characteristic of prostatic incidental adenocarcinoma in contemporary radical cystoprostatectomy specimens. BJU Int 2007;99:554-558.
24 Rivas JG, Gregorio SA, Gómez ÁT, Alvarez-Maestro M, Sebastián JD, Ledo JC: Laparoscopic radical cystectomy with prostate capsule sparing. Initial experience. Cent European J Urol 2016;69:25-31.

25 Saidi A, Nahon O, Daniel L, Lay F, Lechevallier E, Coulange C: Prostate-sparing cystectomy: long-term functional and oncological results in a series of 25 cases. Prog Urol 2004;14:172-177.

-26 Kefer JC, Campbell SC: Current status of prostate-sparing cystectomy. Urol Oncol 2008;26:486-493. 